\title{
Implementation of Sparse Image Acquisition in a Conventional Scanning Transmission Electron Microscope
}

Matt Tanner ${ }^{1}$, Sunghwan Hwang ${ }^{1}$, Volkan Ortalan ${ }^{1}$, Chang Wan Han $^{2}$, Singanallur V Venkatakrishnan ${ }^{2}$ and Charles A Bouman ${ }^{2}$

${ }^{1}$ School of Materials Engineering and Birck Nanotechnology Center, Purdue University, West Lafayette, IN, USA

${ }^{2 .}$ School of Electrical and Computer Engineering, Purdue University, West Lafayette, IN, USA

STEM is one of the foremost methods for nanoscale characterization of materials, but high current densities can cause damage in electron-beam sensitive materials. Here, we report our recent approach to implement a sparse acquisition in STEM mode executed by a random sparse-scan and a signal processing algorithm called model-based iterative reconstruction (MBIR) [1]. In this method, a small portion (such as 5\%) of randomly chosen unit sampling areas (i.e. electron probe positions), which corresponds to pixels of a STEM image of the specimen are scanned with an electron probe to obtain a sparse image. Sparse images are then reconstructed using the MBIR inpainting algorithm to produce an image of the specimen at the original resolution that is consistent with an image obtained using conventional scanning methods. Experimental results for down to $5 \%$ sampling show consistency with the full STEM image acquired by the conventional scanning method.

This reconstruction method was implemented with no hardware modification, on a conventional FEI Titan 80-300 S/TEM. The sample was gold nanoparticles on an amorphous carbon film. The random sparsescan STEM technique is mainly composed of a non-raster scan based image acquisition followed by an image inpainting process to reconstruct the fully sampled image from a partially sampled one. Figure 1 describes the process flow of the method for acquiring a subsampled image and image reconstruction. The ROI of a specimen is expressed as an array of 'discrete' unit sampling areas (figures 1(b) and (d)). In contrast to a conventional STEM, only a small number of unit sampling areas are randomly chosen and exposed to the electron probe during the random sparse-scan (figure 1(d)). Therefore, an image is acquired that has intensities of randomly chosen unit sampling areas, as illustrated in figure 1(e). Then, the sparsely acquired image (figure 1(e)) is used to reconstruct the full image (figure 1(f)) by the MBIR based imageinpainting algorithm. The MBIR [2] approach, used to reconstruct the image, exploits the physics of image formation and information on the noise statistics. MBIR is a very powerful framework for solving data reconstruction problems. Functionally, the algorithm attempts to find the value of each position that matches the data while being constrained by the probability density function of the unknown image.

Figure 2 demonstrates a 10\%, 5\%, and 3\% sampled image, and their corresponding reconstructions. The $10 \%$ and $5 \%$ images are reconstructed with high consistency with the conventional image, although the $5 \%$ image does see blurring around the edge of each particle. The 3\% image is rather suppressed, and sees further blurring of the particle edges. Next, the reconstructed image quality was evaluated by comparing the intensity profile along a line of the sampled, and conventional, images (a $20 \%$ sampled image is included). The difference between $20 \%$ and $10 \%$ sample imaging in terms of particle diameter and number of particles was negligible, being equivalent to up to one place of decimals. There is only a $2 \%$ difference between the 5\% sampling the reference image, and the 3\% sampled image accurately predicts particle diameter with only a $5 \%$ error. These numbers can be further improved by utilizing an online 
reconstruction strategy (as opposed to offline), or utilizing the reconstruction algorithm in real time to optimize the acquisition to focus on important areas of the sample [3].

Ultimately, the random sparse acquisition technique for STEM imaging demonstrates the potential for utilization with beam-sensitive materials. Hardware modifications and software optimization could increase the effectiveness of this technique, lowering the electron dose by up to a factor of 20 . We will discuss the significant potential of the sparse acquisition STEM for low dose imaging and the current limitations of the application of sparse imaging in conventional STEM instruments together with some possible strategies to overcome these shortcomings.

References:

[1] Sauer K and Bouman C, IEEE Trans. Signal Process. 41 (1993) p. 534.

[2] Yu Z et al, IEEE Trans. Image Process. 20 (2011) p. 161.

[3] Godaliyadda G M D, Buzzard G T, and Bouman C A, IEEE Int. Conf. on Acoustics Speech and Signal Processing (2014) p. 1822.

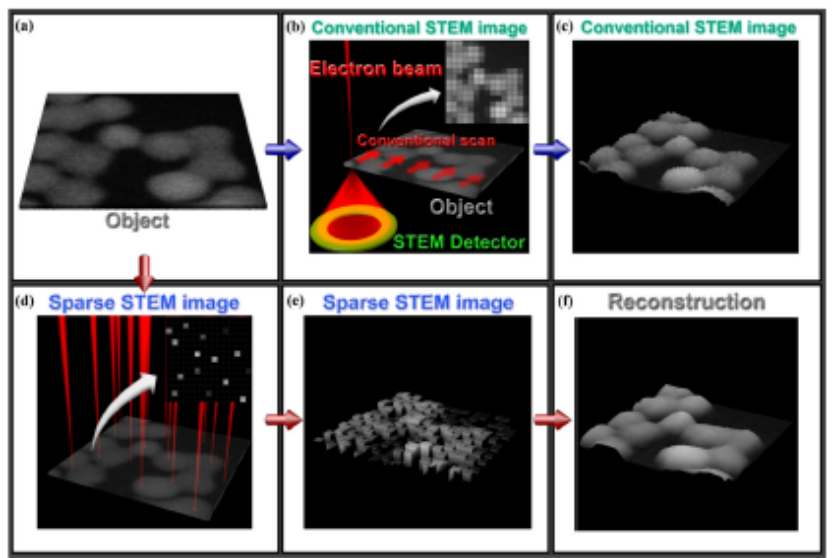

Figure 1. Illustration of conventional STEM imaging (a)-(c), and the process flow of the sparse acquisition STEM technique: (d) Discontinuous random sparse scan. (e) 3D intensity surface plot of the sparsely collected STEM image. (f) 3D intensity surface plot reconstructed using the MBIR technique.

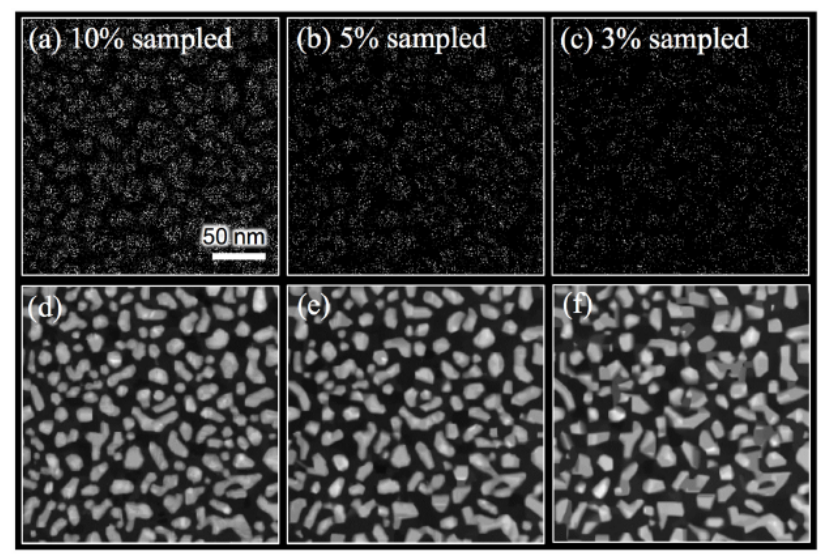

Figure 2. Random sparse images and their reconstructions. The random sparse images with the sampling ratio of 10,5, and 3\% (a)-(c), and their reconstructions (d)-(f) are shown, respectively. 